\title{
Anticipated and zero-lag synchronization in motifs of delay-coupled systems
}

\author{
Claudio R. Mirasso, ${ }^{1}$ Pedro V. Carelli, ${ }^{2}$ Tiago Pereira, ${ }^{3}$ Fernanda S. Matias, ${ }^{4}$ \\ and Mauro Copelli ${ }^{2}$ \\ ${ }^{1}$ Instituto de Física Interdisciplinar y Sistemas Complejos IFISC (CSIC-UIB), Universitat de les Illes Baleares, \\ Campus UIB, E-07122 Palma de Mallorca, Spain \\ ${ }^{2}$ Departamento de Física, Universidade Federal de Pernambuco, Recife, Pernambuco, Brazil \\ ${ }^{3}$ Instituto de Ciências Matemáticas e de Computação, Universidade de São Paulo, São Carlos, São Paulo, \\ Brazil \\ ${ }^{4}$ Departamento de Física, Universidade Federal de Alagoas, Maceió, Alagoas, Brazil
}

(Received 15 May 2017; accepted 23 August 2017; published online 11 October 2017)

\begin{abstract}
Anticipated and zero-lag synchronization have been observed in different scientific fields. In the brain, they might play a fundamental role in information processing, temporal coding and spatial attention. Recent numerical work on anticipated and zero-lag synchronization studied the role of delays. However, an analytical understanding of the conditions for these phenomena remains elusive. In this paper, we study both phenomena in systems with small delays. By performing a phase reduction and studying phase locked solutions, we uncover the functional relation between the delay, excitation and inhibition for the onset of anticipated synchronization in a sender-receiverinterneuron motif. In the case of zero-lag synchronization in a chain motif, we determine the stability conditions. These analytical solutions provide an excellent prediction of the phase-locked regimes of Hodgkin-Huxley models and Roessler oscillators. Published by AIP Publishing.

https://doi.org/10.1063/1.5006932
\end{abstract}

\begin{abstract}
Anticipated and zero-lag synchronization are phenomena that are believed to play important roles in the brain, with evidence suggesting that they occur despite the conductance delay between cortical areas. Both synchronization regimes were reported in numerical simulations of motifs of three-node neuronal systems coupled with delay. Here, we present an analytical approach to these problems by performing a phase reduction leading to a phase oscillator model. Since neurons have firing rates of the order of tens of Hertz and conduction delays are in the range of tens of milliseconds, the regime of small delays (less than the period of free-running units) is justified and simplifies the calculations. In this framework, three regimes are predicted to occur as inhibition is increased in a sender-receiver-interneuron (SRI) motif: delayed synchronization (DS), anticipated synchronization (AS), and finally phase drift. Varying the delay in a chain motif of three mutually connected units, on the other hand, the locking between the central and the outer nodes is shown to be close to either in-phase or antiphase, with instability (phase drift) gaps in-between. The analytical results show very good agreement with numerical simulations of motifs of Hodgkin-Huxley neurons and Roessler oscillators.
\end{abstract}

\section{INTRODUCTION}

The activation of cells in the visual cortex has been observed to occur simultaneously under a visual stimulus. ${ }^{1-3}$ Synchronization of neuronal activity often occurs for long range interactions, having been observed across cortical regions and between cortical and subcortical structures. ${ }^{4-8}$ The outer nodes in this motif act as two populations that synchronize at zero-lag, while the middle one acts as a relay element, which could be another cortical or sub-cortical structure, communicating with the external nodes of the motif. Surprisingly, although these areas are far apart and propagation delays are present, these areas synchronize without any lag. The dynamical mechanisms that compensate the propagation delays are the focus of intense research.

In the presence of transmission delays, anticipated synchronization $^{9-14}$ is another remarkable collective behaviour that is observed in monkeys performing visual discrimination tasks. $^{6,7}$ Multi-electrode recordings showed a dominant directional influence between areas of sensorimotor cortex (as assessed by Granger causality), accompanied by either a negative or a positive phase lag between them. In the striking example found in Brovelli et al., the primary somatosensory cortex (S1) Granger causes the primary motor cortex (M1) while both coherently oscillate in the beta frequency range. ${ }^{6}$ However, the phase of the oscillation of M1 is ahead of that of S1, so, in that sense, M1 anticipates S1. Roughly speaking, when the driven area is ahead of the driver, the phase difference between the areas becomes negative.

The apparent contradiction of having a clear directional influence together with a negative phase difference was investigated numerically on models of two coupled neuronal populations. ${ }^{15-17}$ The numerics suggest that inhibitory conductance in the receiver population can change the dynamics from the delayed synchronization (positive phase difference) to the less intuitive regime of anticipated synchronization (negative phase difference). ${ }^{16,17}$

More recently, the concept of anticipated synchronization $^{9,10}$ was applied to theoretically understand a behavioral neuroscience study. ${ }^{18-20}$ Moreover, at the level of neural circuits, the sign of the lag also plays a major role in learning, 
through the mechanism of spike-timing-dependent plasticity (STDP). ${ }^{21}$ The interplay between STDP and different synchronization regimes has been numerically investigated in a model of neuronal populations. ${ }^{22}$

Up to now, these phenomena have been treated mostly numerically, $8,16-18,22-24$ and a general understanding remains elusive. In this paper, we analytically tackle the phenomena of zero-lag and anticipated synchronization in neuronal motifs of three nodes, focusing on the stability of phase locked solutions. By performing a phase reduction, the stability of phase locked states can be determined in terms of a phase oscillator model. This allows us to obtain a detailed understanding of both anticipated and zero-lag synchronization when the delay is small. Our main contributions are:

(i) We obtain the nonlinear functional relation between excitation and inhibition to have anticipated synchronization. We consider a sender-receiver-interneuron (SRI) motif. The sender and receiver send excitatory synapses, whereas the interneuron inhibits the receiver. In this setting, there is a critical value of inhibition for the transition to anticipated synchronization.

(ii) We obtain the stability conditions for zero-lag synchronization. In a chain motif with delay coupling, our analysis reveals the role of delay in the stability conditions for the ends of the chain to exhibit zero-lag synchronization.

Chaotic oscillators that are phase coherent are also amenable to the treatment of phase reduction. In the present paper, we focus on phase synchronization, in contrast to previous work that addressed stronger forms of synchronization. ${ }^{25-29}$ We probe our predictions in Hodgkin-Huxley model circuits with inhibitory and excitatory transmission delays as well as in circuits of delay-coupled chaotic Roessler oscillators in the phase coherent regime. The analytical predictions show excellent agreement with numerical simulations.

\section{DELAY COUPLED OSCILLATORS}

Consider the $N$ coupled systems with delays due to signal propagation

$$
\dot{\boldsymbol{x}}_{i}(t)=\boldsymbol{f}_{i}\left(\boldsymbol{x}_{i}(t)\right)+\sum_{j} W_{i j} \boldsymbol{h}_{i}\left(\boldsymbol{x}_{i}(t), \boldsymbol{x}_{j}\left(t-\tau_{i j}\right)\right),
$$

where $i=1, \ldots, N, \boldsymbol{f}_{i}$ is the vector field of the isolated system, $\boldsymbol{h}_{i}$ is the coupling function, and $W_{i j}$ is nonzero if $i$ receives a connection from $j$ with weight $W_{i j}$. Here, $\tau_{i j}$ stands for the delays in transmission.

Delay-coupled systems have been thoroughly studied in recent years (see, e.g., Ref. 30 for a thematic issue). The impact of the delay on the dynamics depends on its value as compared to the internal time scale of the system. Interestingly, delay can play two opposite roles impacting the dynamics of a system: on the one hand, it can have a stabilizing role, as e.g., yielding zero-lag synchronization in three delay-coupled chaotic systems ${ }^{31}$ or stabilizing the otherwise periodic dynamics of coupled limit-cycle oscillators, the so-called death by delay. ${ }^{32}$ On the other hand, delayed feedback can destabilize a system, giving rise to chaotic oscillations, as is well known e.g., in one semiconductor laser subject to an optical self-feedback. ${ }^{33}$ Also, in networks of delay coupled oscillators, structural changes that improve the network structure can lead to synchronization loss. ${ }^{34,35}$

\section{A. Phase reduction}

If the isolated dynamics of all systems has a stable periodic orbit $\gamma$, then we can perform a phase reduction near the orbit and obtain the phase description for the coupled oscillator. This approach was introduced previously by different authors. ${ }^{36-39}$ If the $i$ th node has a periodic orbit $\gamma_{i}$, such an orbit can be parametrized by its phase $\gamma_{i}(t)=\gamma_{i}\left(\theta_{i}(t)\right)$. When the coupling is small, the amplitude will only change slightly and all relevant dynamics will be described by the phases $\theta_{i}$ 's. If the periodic orbit emerges from a Hopf bifurcation, the phase description will be given by a Kuramotolike model with delay interaction

$$
\dot{\theta}_{i}=\omega_{i}+\sum_{j \neq i} K_{j i} \sin \left(\theta_{j}(t-\tau)-\theta_{i}(t)\right),
$$

for $i=1, \ldots, N$.

Delay-coupled Kuramoto oscillators were analyzed via analytical methods and numerical simulations for some particular motifs. In rings of unidirectionally and bidirectionally connected elements, in-phase and out-of-phase solutions and their stability were found. ${ }^{40}$ The role of amplitude and phase instabilities of delay-coupled Stuart-Landau oscillators was also studied analytically and numerically. ${ }^{41}$

\section{B. Dynamics near phase locking}

Throughout the manuscript, we are interested in the case when the phases are phase locked

$$
\theta_{i}(t)=\theta_{1}(t)+\phi_{i}
$$

where $\phi_{i}$ is a constant. Along the phase locked solutions, the effect of a time delay on the connection between two elements $j$ and $i$ translates into a constant phase $\delta_{j i}$ shift.

$$
\dot{\theta}_{i}=\omega_{i}+\sum_{j \neq i} K_{j i} \sin \left(\theta_{j}-\theta_{i}-\delta_{j i}\right), \quad i=1, \ldots, N,
$$

where $\omega_{i}$ are the natural frequencies, $\delta_{j i}=\omega_{j} \tau_{j i}$, and $K_{j i}$ are the coupling strengths. The coupling values can be either positive or negative, which e.g., in neuronal systems, would represent excitatory or inhibitory synaptic couplings.

\section{The motifs}

We will focus on two $W_{i j}$ configurations which correspond to 3-node motifs of particular relevance in neuroscience. Motifs are subgraphs of larger networks that occur beyond chance, and are thus regarded as building blocks of complex networks. ${ }^{42}$

The first motif will be referred to as sender-receiverinterneuron (SRI), and is shown in Fig. 1(a). The receiver is mutually connected with the interneuron, being also driven by the sender. The sender and the receiver are excitatory, whereas the interneuron is inhibitory. The SRI motif is 
(a)
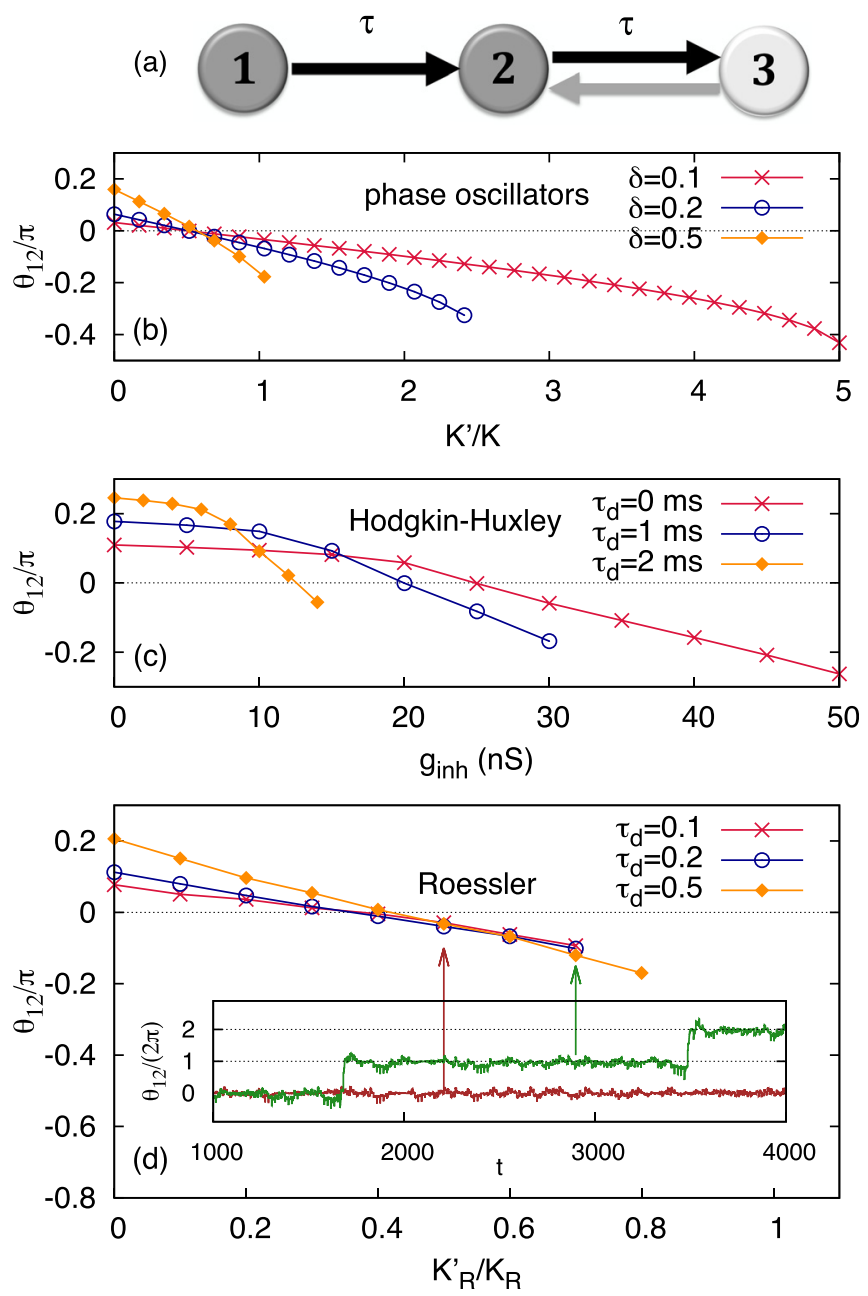

FIG. 1. Dependence of the sender-receiver phase $\theta_{12}^{*}$ on the inhibitory (negative) coupling (exact for the phase model and time-averaged for the Roessler model) shows a transition from delayed $\left(\theta_{12}^{*}>0\right)$ to anticipated $\left(\theta_{12}^{*}<0\right)$ synchronization, and then to phase drift. (a) Representation of the motif. (b) Phase-oscillators, considering $\delta=\delta^{\prime}$. (c) Hodgkin-Huxley neuron models. (d) Roessler systems coupled in the $x$ and $y$ variables with a delay $\tau_{d}$. Inset: Phase difference $\theta_{12}$ as a function of time.

ubiquitous in different regions of the vertebrate brain, such as the thalamus, ${ }^{43,44}$ spinal cord ${ }^{45}$ and nuclei involved with song production in the bird brain. ${ }^{46}$ Sporns and Kötter, ${ }^{47}$ as well as Qian et al., ${ }^{48}$ have also shown that this motif is particularly prevalent in the connectome of the nematode $C$. elegans.

The second motif, we will study, is a chain of mutually connected units ("V-motif"), as shown in Fig. 4. According to Sporns and Kötter, ${ }^{47}$ this is the most prevalent motif in the macaque and cat cortices. Moreover, it is a good model of cortico-thalamo-cortical loops, the outer elements representing different cortical regions and the central element representing a thalamic region. ${ }^{8}$

\section{MODELS}

In this section, we introduce the Hodgkin-Huxley model (Sec. III A), which admits a phase reduction. ${ }^{49}$ This is a natural setting for our analysis. Chaotic oscillators also admit a phase reduction when the dynamics is phase coherent. This is the case of the Roessler attractor, which we will introduce in Sec. III B. In this case, amplitudes and phases are coupled. The amplitudes act as effective noise in the phase dynamics. ${ }^{50}$ Since the anticipated synchronization corresponds to an exponentially stable dynamics, it will persist under small noise.

\section{A. Hodgkin-Huxley model}

The Hodgkin-Huxley model ${ }^{51}$ consists of four differential equations describing the evolution of the membrane potential and the currents flowing across a patch of an axonal membrane 52

$$
\begin{aligned}
C_{m} \frac{d V}{d t}= & \bar{G}_{N a} m^{3} h\left(E_{N a}-V\right)+\bar{G}_{K} n^{4}\left(E_{K}-V\right) \\
& +G_{m}\left(V_{\text {rest }}-V\right)+I_{c}+\sum I_{\text {syn }} \\
& \frac{d x}{d t}=\alpha_{x}(V)(1-x)-\beta_{x}(V) x .
\end{aligned}
$$

Here, $V$ is the membrane potential and $x \in\{h, m, n\}$ are the gating variables for sodium ( $h$ and $m$ ) and potassium $(n)$. The capacitance of a $30 \times 30 \times \pi \mu \mathrm{m}^{2}$ equipotential patch of membrane is $C_{m}=9 \pi \mu \mathrm{F}^{52} E_{N a}=115 \mathrm{mV}, E_{K}=-12 \mathrm{mV}$ and $V_{\text {rest }}=10.6 \mathrm{mV}$ are the reversal potentials of $\mathrm{Na}^{+}, \mathrm{K}^{+}$ and leakage currents, respectively. The maximal conductances are $\bar{G}_{N a}=1080 \pi \mathrm{mS}, \bar{G}_{K}=324 \pi \mathrm{mS}$ and $G_{m}=2.7 \pi$ $\mathrm{mS}$, respectively. $I_{s y n}$ accounts for the chemical synapses arriving from other neurons and $I_{c}=280 \mathrm{pA}$ accounts for an external constant current. The voltage-dependent rate variables in the Hodgkin-Huxley model are the standard values. ${ }^{53}$

The synaptic current at each synapse is given by

$$
I_{\text {syn }}(t)=g_{\text {syn }} \sum_{\text {spikes }} \alpha\left(t-t_{\text {spike }}-\tau_{d}\right)\left(E_{\text {syn }}-V\right),
$$

where $V$ is the postsynaptic potential, $g_{s y n}$ is the maximal conductance and $E_{\text {syn }}$ is the reversal potential. We use $E_{\text {exc }}$ $=60 \mathrm{mV}$ and $E_{i n h}=-20 \mathrm{mV}$, which are modeled as AMPA and $\mathrm{GABA}_{A}$ mediated synapses, respectively. The internal sum is extended over all the presynaptic spikes occurring at $t_{\text {spike. }}$ The delay in the coupling $\tau_{d}$ arises from the finite conduction velocity of axons, and is accounted by the latency time in the $\alpha$ function, which models postsynaptic conductance changes as follows:

$$
\alpha(t)=\frac{\Theta(t)}{\tau_{-}-\tau_{+}}\left(\exp \left(-t / \tau_{-}\right)-\exp \left(-t / \tau_{+}\right)\right),
$$

where $\Theta$ is the Heaviside function. The parameters $\tau_{-}$and $\tau_{+}$stand, respectively, for the decay and rise times of the function and determine the duration of the synaptic response.

The excitatory conductances and decay and rise times for the three mutually coupled neurons are ("V-motif" of Sec. IVE), respectively, $\quad g_{\text {exc }}=20 \mathrm{nS}, \quad \tau_{-}=4.0 \mathrm{~ms}$ and $\tau_{+}=0.1 \mathrm{~ms}$. For the motif with three neurons in a senderreceiver-interneuron configuration (SRI motif of Sec. IV A), we use $g_{\text {exc }}=10 \mathrm{nS}, \tau_{-}=6.0 \mathrm{~ms}$ and $\tau_{+}=0.1 \mathrm{~ms}$.

For the three excitatory mutually coupled neurons (Sec. IVE), we employ current-based synapses, by fixing $\left(E_{s y n}-V\right)$ $=1 \mathrm{mV}$ in Eq. (7). It is worth mentioning that qualitatively, similar results are obtained with conductance-based coupling models. 


\section{B. Roessler model}

The equations for the coupled Roessler system are given by

$$
\begin{aligned}
& \dot{x}_{i}=-y_{i}-z_{i}+\sum_{j \neq i} k_{j i}\left[x_{j}\left(t-\tau_{d}\right)-x_{i}(t)\right] \\
& \dot{y}_{i}=x_{i}+a y_{i}+\sum_{j \neq i} k_{j i}\left[y_{j}\left(t-\tau_{d}\right)-y_{i}(t)\right] \\
& \dot{z}_{i}=b+z_{i}\left(x_{i}-c\right),
\end{aligned}
$$

where $a=0.2, b=0.2$ and $c=5.7$ for all simulations. With these parameter values, the behaviour of the free-running oscillators is chaotic. The parameter $\tau_{d}$ accounts for the delay in the coupling. The coupling term $k_{i j}(i \neq j)$ can be either positive or negative. We set al.l the nonzero positive $k_{i j}=K_{R}=0.05$ in Sec. IV A, whereas $k_{i j}=K_{R}=0.01$ in Sec. IV E. For all cases, we varied $K_{R}$ between 0.01 and 0.1 and found similar results, except in the motif of Sec. IV E, for which $K_{R}=0.1$ was already too strong, eventually leading to the death-by-delay phenomenon for specific delay times. The phase of each Roessler system was defined as $\theta_{i}=\tan ^{-1}\left(y_{i} / x_{i}\right)$ if $x_{i}>0$ and $\theta_{i}=\tan ^{-1}\left(y_{i} / x_{i}\right)+\pi$, if $x_{i}<0 .{ }^{50,54,55}$

\section{RESULTS}

To study phase-locked solutions and their stability, we define the three relative phases

$$
\theta_{i j} \equiv \theta_{i}-\theta_{j}, \quad i, j \in\{1,2,3\}, i<j,
$$

and compare their stationary values [analytically predicted by Eq. (4)] with those obtained from numerical simulations of the Hodgkin-Huxley and Roessler models. Non-zero stationary values of $\theta_{i j}$ imply a phase lag.

\section{A. Sender-Receiver-Interneuron (SRI) motif}

Here, we show that the phase oscillator model in the SRI motif [Fig. 1(a)] correctly predicts the transition between delayed synchronization (DS) and anticipated synchronization (AS), each regime characterized by a different sign of $\theta_{12}^{*}$ (positive for DS, negative for AS). Specifically, we obtain the analytical expression for the dependence of the AS-DS transition on the inhibitory and excitatory coupling strengths as well as on the delay [Eq. (12), which is the main result of this section].

In the SRI circuit (which corresponds to motif 7 of Ref. 42 or motif 4 of Ref. 47), a sender node feeds a receiver that is coupled to a third node, receiving from it an inhibitory feedback [Fig. 1(a)]. Previous numerical results with Hodgkin-Huxley neurons had shown that a continuous transition occurs between anticipated and delayed anticipation when the inhibitory coupling changes, ${ }^{16}$ but an analytical explanation was still lacking. We show below that the analytical prediction of the phase oscillators is well matched by simulations of both $\mathrm{HH}$ and Roessler motifs.

We choose for all frequencies, the same $\omega_{i}=\omega$ and couplings $K_{12}=K_{23}=K>0, K_{21}=0$ and $K_{32}=-K^{\prime}<0$, $\delta_{12}=\delta_{23}=\delta, \delta_{32}=\delta^{\prime}$, focusing on the dependence of the phase difference between the sender and the receiver $\left(\theta_{12}^{*}\right)$ as a function of the inhibitory coupling $K^{\prime}$.

\section{B. Phase locking conditions}

When imposing the phase locking conditions, Eq. (3), we have that $\theta_{12}^{*}=-\phi_{2}\left(K, K^{\prime}, \delta, \delta^{\prime}\right)$, so we readily obtain $\dot{\theta}_{2}=\dot{\theta}_{1}$, implying that $K \sin \left(\theta_{12}^{*}-\delta\right)-K^{\prime} \sin \left(\theta_{32}^{*}-\delta^{\prime}\right)=0$ together with $\dot{\theta}_{3}=\dot{\theta}_{1}$, yielding $K \sin \left(\theta_{23}^{*}-\delta\right)=0$. Therefore, the conditions on the phase differences are

$$
\begin{gathered}
\theta_{23}^{*}=\delta, \\
\theta_{12}^{*}=\delta-\arcsin \left(\frac{K^{\prime}}{K} \sin \left(\delta+\delta^{\prime}\right)\right),
\end{gathered}
$$

where we note that

$$
\frac{K^{\prime}}{K}\left|\sin \left(\delta+\delta^{\prime}\right)\right| \leq 1
$$

is a necessary condition for the phase-locked solution between the sender and the receiver to exist.

Anticipated Synchronization: When anticipated synchronization takes place, the phase $\theta_{2}$ of node two is ahead of the phase $\theta_{1}$. Hence, in this construction, $\theta_{12}<0$. From Eq. (12), we obtain a condition for anticipated synchronization. In particular, for small delays $\delta=\delta^{\prime} \ll 1$, we obtain that the transition occurs when

$$
K^{\prime}>\frac{K}{2}
$$

so, anticipated synchronization takes place when the inhibition is strong enough.

\section{Stability of phase-locking}

In the limit $\delta^{\prime}=\delta \ll 1$, the delay equations inherit the stability of the delay-free system. ${ }^{56}$ We can obtain the linear stability properties of the phase-locked solution by analyzing the growth of perturbations $\xi_{j}(t), j=2,3$, defined as:

$$
\theta_{j}(t)=\theta_{1}(t)+\phi_{j}+\xi_{j}(t)
$$

Introducing the notation $\boldsymbol{\xi}(t)=\left(\xi_{2}(t), \xi_{3}(t)\right)^{T}$, a Taylor expansion for small $\xi_{j}$ yields

$$
\dot{\xi}(t)=A \xi(t)+B \xi(t-\tau)
$$

where we ignored higher-order terms in $\xi$. Moreover,

$$
A=\left(\begin{array}{cc}
-K \cos \left(\delta-\theta_{12}^{*}\right)+K^{\prime} \cos \left(\delta-\theta_{23}^{*}\right) & 0 \\
0 & -K
\end{array}\right)
$$

and

$$
B=\left(\begin{array}{cc}
0 & -K^{\prime} \cos \left(\delta-\theta_{23}^{*}\right) \\
K & 0
\end{array}\right)
$$

Next, consider the characteristic equation $p(\lambda)=$ det $\left(\lambda I-A-B e^{\lambda \tau}\right)$. For small delays $\tau \ll 1$, we have $p(\lambda)=\operatorname{det}(\lambda I-A-B)+O(\tau)$, as the eigenvalues of $A+B$ 
are simple. Indeed, an inspection shows that the spectrum of $A+B$ has only negative real part as long as

$$
2 K-K^{\prime}>0 \text {. }
$$

Hence, Eqs. (14) and (19) provide the conditions for anticipated synchronization in the small- $\delta$ limit.

\section{AS in Models: Numerics vs. predictions}

We observe a transition from delayed to anticipated synchronization, when $\theta_{12}^{*}$ changes sign for increasing $K^{\prime}$ [Fig. 1(b)]. As can be seen from Eq. (12), this transition happens at $K^{\prime} \approx K / 2$, for $\delta=\delta^{\prime} \ll 1$. Increasing $K^{\prime}$ further, condition (13) breaks down and phase locking is no longer a solution.

The same trend is also observed in both the HodgkinHuxley [Fig. 1(c)] and the Roessler models [Fig. 1(d)]. In both cases, the transition from DS to AS occurs, and eventually a phase drift regime is reached when the inhibitory (negative) coupling is increased. Also, the slope of the curves increases with the delay. In particular, for the Roessler system, by increasing the negative coupling $K_{R}^{\prime}$, initially an imperfect phase synchronization is observed [see the inset of Fig. 1(d)] and then synchronization becomes unstable and a phase drift regime is reached.

In Fig. 2, we show time traces for the Hodgkin-Huxley model. Panels (a) and (b) correspond to phase-locked solutions, where the receiver lags the sender (panel a) or the
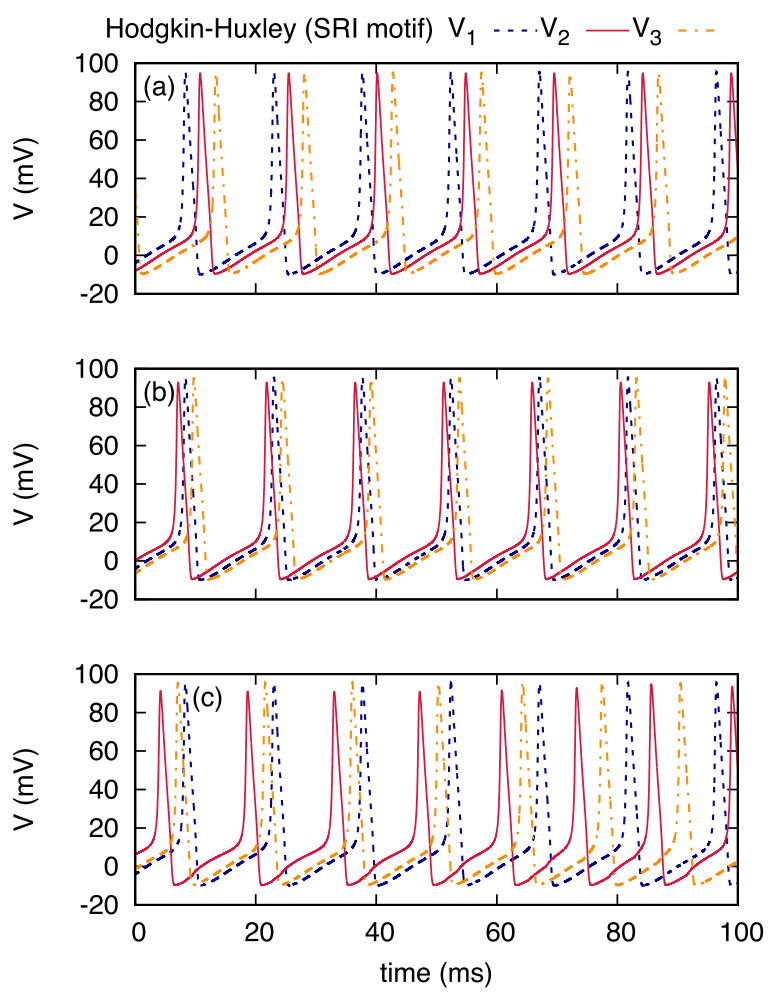

FIG. 2. Time traces of the membrane potentials of the Hodgkin-Huxley units [Eq. (5)] in the SRI motif [Fig. 1(a)] show three different regimes as inhibition increases: DS, AS and phase drift. $\tau_{d}=1 \mathrm{~ms}$ and (a) $g_{\text {inh }}=5 \mathrm{nS}$ (delayed synchronization), (b) $g_{\text {inh }}=25 \mathrm{nS}$ (anticipated synchronization) and (c) $g_{\text {inh }}=40 \mathrm{nS}$ (phase drift). See also Fig. 1(c). receiver anticipates the sender (panel b). Finally, in panel (c), the inhibitory conductance is large enough that the phaselocked solution is lost and a phase drift regime is observed.

The time series of $x$ variables of the Roessler sender and receiver units are shown in Fig. 3. For weak inhibition [Figs. 3(a) and 3(b)], the sender leads the receiver in phase on average, although with fluctuations. With increasing inhibitory coupling, we observe a transition from delayed to anticipated synchronization, again on average and with fluctuations [see Figs. 3(c) and 3(d)]. Finally, for larger values of the inhibitory coupling, phase locking is lost and a phase drift regime ensues, as can be seen in Figs. 3(e) and 3(f).

\section{E. Chain of three mutually delay-coupled systems ("V-motif")}

We now analyze the case of a chain of three mutually coupled phase-oscillators [Fig. 4(a), which is motif 8 in Ref. 42 and motif 9 in Ref. 47]. The phase oscillator model predicts the conditions to find synchronized periodic solutions, in particular, zero-lag synchronization between the "outers" (nodes 1 and 3 in Fig. 4). It also predicts the cases where the middle element will be synchronized in phase or in antiphase with the other elements. As we discussed for the AS, for small delays, the delayed systems inherit the stability of the not delayed systems. So, we will only perform the analysis of the stability of the phase-locked solutions.

Roessler (SRI motif)
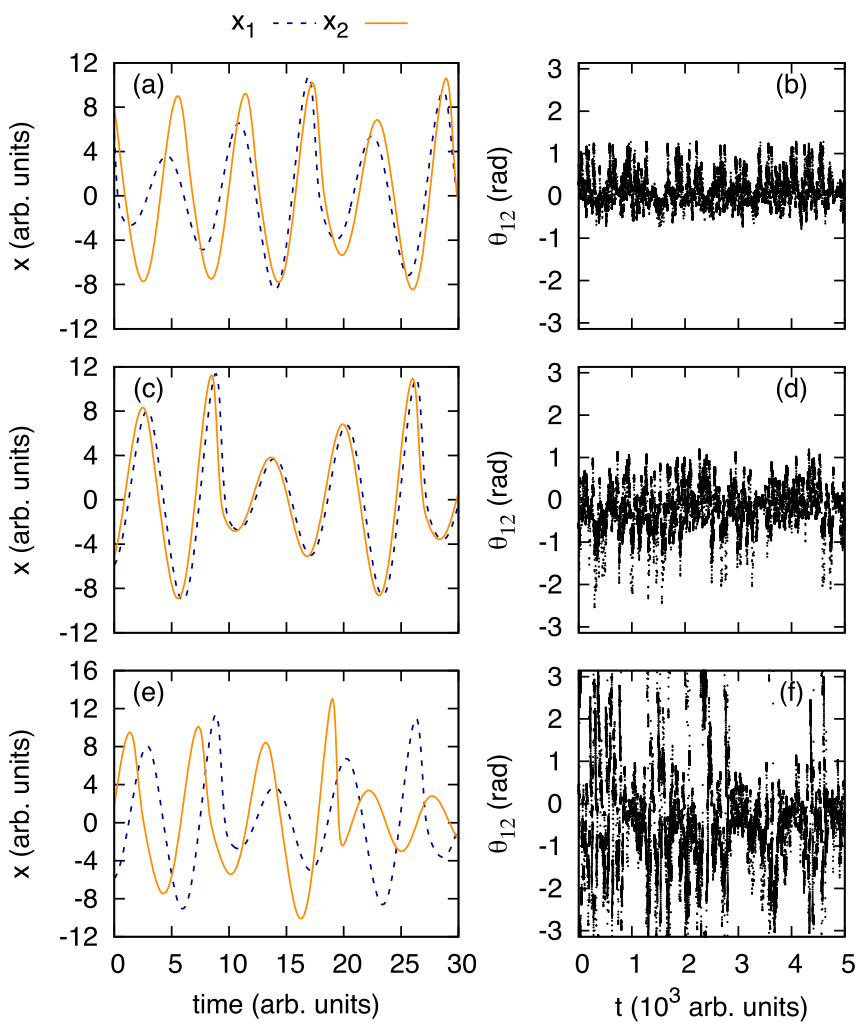

FIG. 3. In the SRI motif [Fig. 1(a)], time traces of the $x$ variables of the Roessler units [Eq. (9)], as well as $\theta_{12}$, show three different regimes as inhibition increases: DS, AS and phase drift. $\tau_{d}=0.1$ and (a) and (b) $K_{R}^{\prime} / K_{R}=0.2$ (delayed synchronization); (c) and (d) $K_{R}^{\prime} / K_{R}=0.6$ (anticipated synchronization), and (e) and (f) $K_{R}^{\prime} / K_{R}=0.9$ (phase drift). Compare with Fig. 1(d). 
(a)
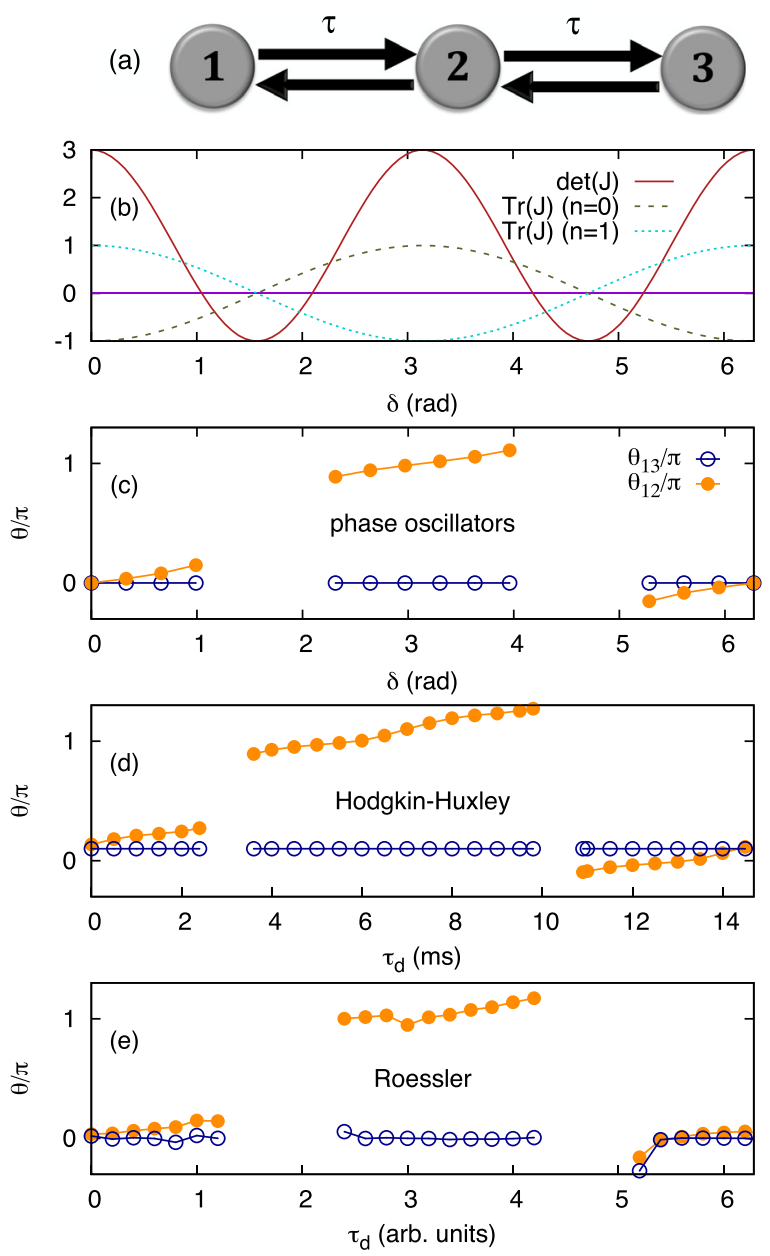

FIG. 4. Phase-locked solutions for the "V-motif" as a function of the delay/ phase shift. Regions where the central node is close to in-phase or anti-phase synchronization with the outers are separated by unstable regions of phase drift. (a) Representation of the motif. (b) Stability of the "outers-in-phase" solutions of the bidirectional chain ("V-motif") according to Eqs. (20) and (21). Dependence of $\operatorname{det}(J)$ (solid) and $\operatorname{Tr}(J)$ (dashed) on the phase difference $\delta$ in the coupling for $K=1$. Dependence of the stable phase-locked fixed point (filled circles for $\theta_{12}^{*}$, empty circles for $\theta_{13}^{*}$, exact for the phase model, time-averaged for the Roessler model) as a function of the phase $\delta$ or delay $\tau_{d}$ in the coupling for the (c) phase oscillators, (d) Hodgkin-Huxley model and (e) Roessler system.

For this motif, we take the most symmetric case $\omega_{i}=\omega, K_{12}=K_{21}=K_{23}=K_{32}=K>0, \quad K_{13}=K_{31}=0$ and $\delta_{2,1}=\delta_{1,2}=\delta_{3,2}=\delta_{2,3}=\delta \geq 0$. In this case, the appropriate variables are $\theta_{12}$, the phase difference between an outer node and the central one, and $\theta_{13}$, the phase difference between the two outer nodes [see Eq. (10)]. After simple algebraic manipulations, one obtains two fixed point solutions. The first one is

$$
\begin{gathered}
\theta_{13}^{*}=0 \\
\theta_{12}^{*}=\arctan (\tan (\delta) / 3)-n \pi, \quad n=0,1
\end{gathered}
$$

which corresponds to a zero-lag synchronization between the outer oscillators ("outers-in-phase"), and a nonzero phaselocking solution between the central and each outer node. The second solution is

$$
\begin{gathered}
\theta_{13}^{*}=(2 n-1) \pi \\
\theta_{12}^{*}=n \pi-\delta, \quad n=0,1
\end{gathered}
$$

and corresponds to anti-phase synchronization between the outer oscillators ("outers-out-of-phase").

From the Jacobian matrix, we obtain

$$
\begin{aligned}
\operatorname{Tr}(J)= & -2 K \cos \delta\left[\cos \theta_{12}^{*}+\cos \left(\theta_{13}^{*}-\theta_{12}^{*}\right)\right] \\
\operatorname{det}(J)= & K^{2}\left[2 \cos \theta_{12}^{*} \cos \delta \cos \left(\theta_{13}^{*}-\theta_{12}^{*}-\delta\right)\right. \\
& \left.+\cos \left(\theta_{13}^{*}-\theta_{12}^{*}+\delta\right) \cos \left(\theta_{12}^{*}+\delta\right)\right] .
\end{aligned}
$$

The "outers-out-of-phase" solution has $\operatorname{Tr}(J)=0$, but we have numerically verified using Eq. (4) that it is never asymptotically stable. The stability of the "outers-in-phase" solutions is shown in Fig. 4(b) for positive $K$ (in particular, and without loss of generality, $K=1)$ : the sign of $\operatorname{det}(J)$ changes at $\tan ^{2} \delta=3$, so that these solutions are unstable in the intervals $\pi / 3<\delta<2 \pi / 3$ and $4 \pi / 3<\delta<5 \pi / 3$. In its stable region, $n=0$ is the attractor for $\delta<\pi / 3$ or $\delta>5 \pi / 3$, whereas $n=1$ is the attractor for $2 \pi / 3<\delta<4 \pi / 3$ [see Eq. (21)].

The numerical results for three mutually coupled Hodgkin-Huxley models with excitatory chemical synapses and conduction axonal delays [Fig. 4(d)] show qualitatively good agreement with the analytical predictions of the phase model. Figures 5(a)-5(c) correspond to delays $\tau_{d}=0,3$ and
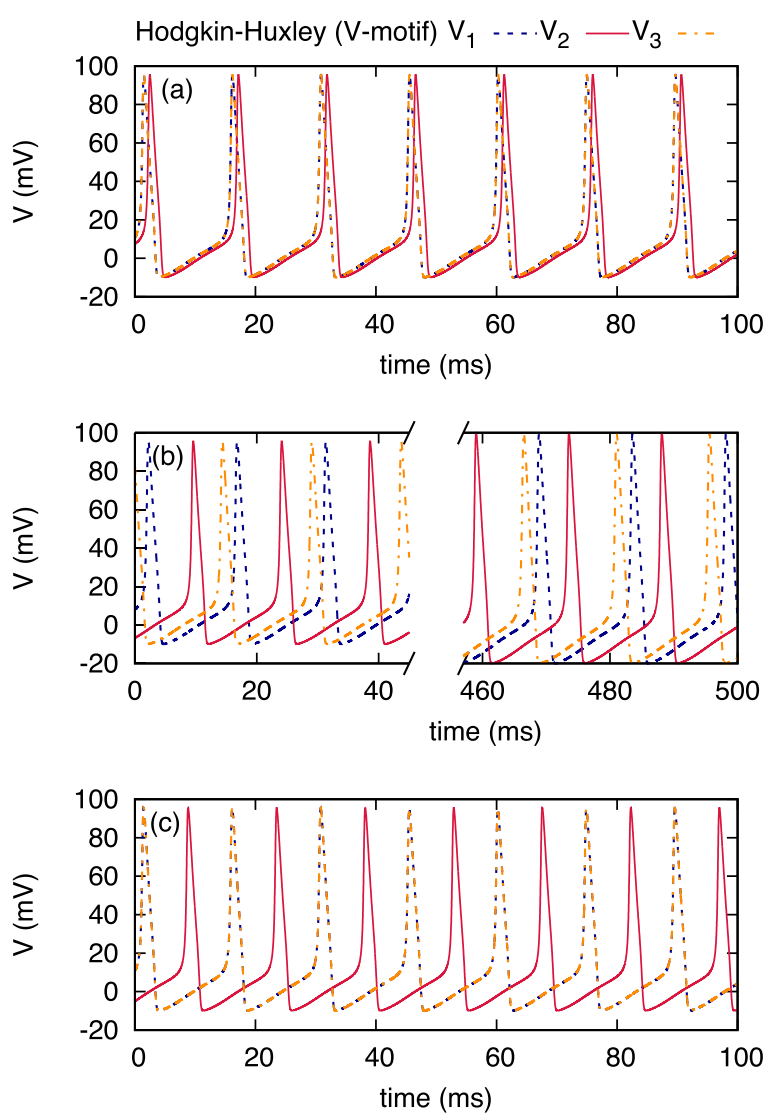

FIG. 5. Time traces of the membrane potentials of the Hodgkin-Huxley units [Eq. (5)] in the V-motif [Fig. 4(a)] illustrate the three scenarios identified in Fig. 4: (a) all nodes close to in-phase sync $\left(\tau_{d}=0 \mathrm{~ms}\right)$, (b) phase drift $\left(\tau_{d}=3 \mathrm{~ms}\right)$, and (c) outers in phase with the central element in anti-phase $\left(\tau_{d}=6 \mathrm{~ms}\right)$. Compare with Fig. 4(d). 
$6 \mathrm{~ms}$, located, respectively, in the in-phase, phase-drift and anti-phase regions of Fig. 4(d). Note in Fig. 5(b) that a long time series is needed to highlight the slow phase-drift regime.

In Fig. 4(e), we show numerical results for three mutually delay-coupled chaotic Roessler systems. From the numerical simulations, we find regions where phase synchronization is stable, and others, where there is no locking. These results agree remarkably well with the analytical predictions of the phase model. As is occurring for the Hodgkin-Huxley model, for small $\tau_{d}$, all three Roessler units are phase synchronized near zero-lag [see Figs. 6(a) and 6(b)]. Note, however, that the central element slightly lags behind the outer ones [Figs. 4(e) and 6(b)]. With increasing delay, we numerically find (as the phase-oscillator model predicts) a region with no phaselocking solution, which can be clearly seen in Figs. 6(c) and 6(d). For $\tau_{d}$ of the order of half of the mean free-running period of the oscillator, we find identical synchronization (phase and amplitude) between the outer nodes, while the central one oscillates in anti-phase with them, although with fluctuations [see Figs. 6(e) and 6(f)].

\section{DISCUSSION}

We have studied motifs of coupled oscillators with delayed interaction. By restricting our attention to phase locked solutions and small delays, we obtained analytical predictions for the stability of the phase locked solutions.
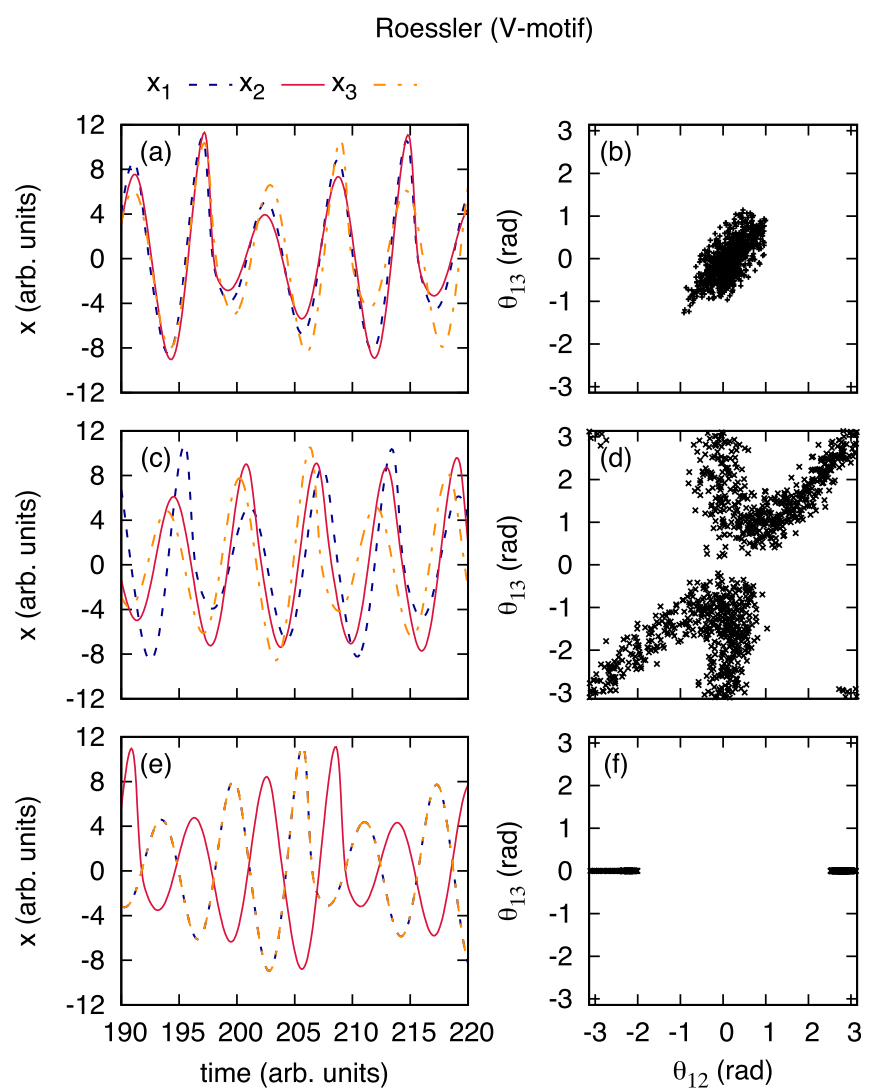

FIG. 6. In the V-motif [Fig. 4(a)], time traces of the $x$ variables of the Roessler units Eq. (9), as well as $\left(\theta_{12}, \theta_{13}\right)$ projections of long time series (2000 time units), illustrate three different regimes: (a) and (b) all in phase ( $\left.\tau_{d}=0.2\right)$; (c) and (d) phase drift $\left(\tau_{d}=2.0\right)$; and (e) and (f) outers in phase with the middle element in anti-phase $\left(\tau_{d}=3.0\right)$. Compare with Fig. 4(e).
This analysis provides good predictions for the numerical simulations of motifs, whose nodes are described by Hodgkin-Huxley or Roessler equations. Until now, the emergence of AS in the SRI motif had only been numerically explored.

We have applied the phase-oscillator approach to small/ moderate coupling strengths and delays up to the characteristic oscillating period of the systems. This seems to be the more relevant scenario in the context of neuronal microcircuits, where neurons have firing rates on the order of tens of Hertz and delays are in the range of tens of milliseconds or less.

If the systems are non-identical or have delays in the transmission, the complete synchronization manifold is not invariant. Because we focused on small delays, the orbits of the coupled system will enter a neighborhood of the synchronization manifold. The stability and the size of the basin of attraction can also be given. ${ }^{57}$ When the coupling increases and the systems are slightly non-identical, a transition to lag synchronization in the amplitudes may also be observed. ${ }^{58}$

Whether the technique is useful for larger delay times remains to be studied. Furthermore, in real systems, the effective phase $\delta$ and the frequency $\omega$ depend on the strength $K$, an effect that, for simplicity, we neglected in this study. It is worth exploring whether, at least for some particular systems, this relationship can be self-consistently deduced.

\section{ACKNOWLEDGMENTS}

We thank enlightening discussions with P. Colet, I. Fischer, E. Hernández-García and R. Vicente. We acknowledge financial support from Brazilian agencies CAPES (PVE Grant No. 88881.068077/2014-01), CNPq (Grant No. 310712/ 2014-9), and FACEPE (Grant No. 0826-1.05/15), as well as Spanish Ministerio de Economía y Competitividad (MINECO) through the project TEC2016-80063-C3-3-R. MC was partially funded by Research, Innovation and Dissemination Center for Neuromathematics (FAPESP Grant No. 2013/07699-0, S. Paulo Research Foundation). T.P. was funded by Centro de Ciências Matemáticas Aplicadas à Indústria (CeMEAI), FAPESP Grant No. 2013/07375-0.

${ }^{1}$ C. M. Gray, P. Konig, A. K. Engel, and W. Singer, "Oscillatory responses in cat visual cortex exhibit intercolumnar synchronization which reflects global stimulus properties," Nature 338, 334-337 (1989).

${ }^{2}$ A. K. Engel, A. K. Kreiter, P. Konig, and W. Singer, "Synchronization of oscillatory neuronal responses between striate and extrastriate visual cortical areas of the cat," Proc. Natl. Acad. Sci. U.S.A. 88, 6048-6052 (1991).

${ }^{3} \mathrm{P}$. Tiesinga, J. M. Fellows, and T. Sejnowski, "Regulation of spike timing in visual cortical circuits," Nat. Rev. Neurosci. 9, 97-109 (2008).

${ }^{4}$ F. Varela, J.-P. Lachaux, E. Rodriguez, and J. Martinerie, "The brainweb: Phase synchronization and large-scale integration," Nat. Rev. Neurosci. 2, 229-239 (2001).

${ }^{5}$ E. Rodriguez, N. George, J.-P. Lachaux, J. Martinerie, B. Renault, and F. J. Varela, "Perception's shadow: Long-distance synchronization of human brain activity," Nature 397, 430-433 (1999).

${ }^{6}$ A. Brovelli, M. Ding, A. Ledberg, Y. Chen, R. Nakamura, and S. L. Bressler, "Beta oscillations in a large-scale sensorimotor cortical network: Directional influences revealed by granger causality," Proc. Natl. Acad. Sci. U.S.A. 101, 9849-9854 (2004).

${ }^{7}$ R. Salazar, N. Dotson, S. Bressler, and C. Gray, "Content-specific frontoparietal synchronization during visual working memory," Science 338, 1097-1100 (2012). 
${ }^{8}$ R. Vicente, L. L. Gollo, C. R. Mirasso, I. Fischer, and G. Pipa, "Dynamical relaying can yield zero time lag neuronal synchrony despite long conduction delays," Proc. Natl. Acad. Sci. U.S.A. 105, 17157-17162 (2008).

${ }^{9}$ H. U. Voss, “Anticipating chaotic synchronization,” Phys. Rev. E 61, 5115 (2000).

${ }^{10} \mathrm{H}$. U. Voss, "Dynamic long-term anticipation of chaotic states," Phys. Rev. Lett. 87, 014102 (2001).

${ }^{11}$ C. Masoller and D. H. Zanette, "Anticipated synchronization in coupled chaotic maps with delays," Phys. A 300, 359-366 (2001).

${ }^{12}$ S. Sivaprakasam, E. M. Shahverdiev, P. S. Spencer, and K. A. Shore, "Experimental demonstration of anticipating synchronization in chaotic semiconductor lasers with optical feedback," Phys. Rev. Lett. 87, 154101 (2001).

${ }^{13}$ T. Pyragiene and K. Pyragas, "Anticipating chaotic synchronization via act-and-wait coupling,” Nonlinear Dyn. 79, 1901-1910 (2015).

${ }^{14}$ Y. Hayashi, S. J. Nasuto, and H. Eberle, "Renormalized time scale for anticipating and lagging synchronization," Phys. Rev. E 93, 052229 (2016).

${ }^{15}$ M. Ciszak, O. Calvo, C. Masoller, C. R. Mirasso, and R. Toral, "Anticipating the response of excitable systems driven by random forcing," Phys. Rev. Lett. 90, 204102 (2003).

${ }^{16}$ F. S. Matias, P. V. Carelli, C. R. Mirasso, and M. Copelli, "Anticipated synchronization in a biologically plausible model of neuronal motifs," Phys. Rev. E 84, 021922 (2011).

${ }^{17}$ F. S. Matias, L. L. Gollo, P. V. Carelli, S. L. Bressler, M. Copelli, and C. R. Mirasso, "Modeling positive granger causality and negative phase lag between cortical areas," NeuroImage 99, 411-418 (2014).

${ }^{18}$ H. U. Voss and N. Stepp, "A negative group delay model for feedbackdelayed manual tracking performance,” J. Comput. Neurosci. 41, 295-304 (2016).

${ }^{19} \mathrm{~N}$. Stepp, “Anticipation in feedback-delayed manual tracking of a chaotic oscillator,” Exp. Brain Res. 198, 521-525 (2009).

${ }^{20}$ N. Stepp and M. T. Turvey, "Anticipation in manual tracking with multiple delays,” J. Exp. Psychol.-Hum. Percept. Perform. 43, 914 (2017).

${ }^{21} \mathrm{G}$. Bi and M. Poo, "Synaptic modifications in cultured hippocampal neurons: Dependence on spike timing, synaptic strength, and postsynaptic cell type," J. Neurosci. 18, 10464-10472 (1998).

${ }^{22}$ F. S. Matias, P. V. Carelli, C. R. Mirasso, and M. Copelli, "Self-organized near-zero-lag synchronization induced by spike-timing dependent plasticity in cortical populations," PloS One 10, e0140504 (2015).

${ }^{23}$ L. L. Gollo, C. R. Mirasso, and A. E. P. Villa, "Dynamic control for synchronization of separated cortical areas through thalamic relay," NeuroImage 52, 947-955 (2010).

${ }^{24}$ L. L. Gollo, C. R. Mirasso, M. Atienza, M. Crespo-Garcia, and J. L. Cantero, "Theta band zero-lag long-range cortical synchronization via hippocampal dynamical relaying," PloS One 6, el7756 (2011).

${ }^{25}$ S. Boccaletti, J. Kurths, G. Osipov, D. L. Valladares, and C. S. Zhou, "The synchronization of chaotic systems," Phys. Rep. 366, 1-101 (2002).

${ }^{26} \mathrm{C}$. Mendoza, S. Boccaletti, and A. Politi, "Convective instabilities of synchronization manifolds in spatially extended systems," Phys. Rev. E 69, 047202 (2004).

${ }^{27}$ K. Pyragas and T. Pyragiene, "Extending anticipation horizon of chaos synchronization schemes with time-delay coupling," Philos. Trans. R. Soc. A 368, 305-317 (2010).

${ }^{28}$ E. M. Shahverdiev, S. Sivaprakasam, and K. A. Shore, "Lag synchronization in time-delayed systems," Phys. Lett. A 292, 320-324 (2002).

${ }^{29}$ A. Englert, S. Heiligenthal, W. Kinzel, and I. Kanter, "Synchronization of chaotic networks with time-delayed couplings: An analytic study," Phys. Rev. E 83, 046222 (2011).

${ }^{30}$ V. Flunkert, I. Fischer, and E. Schöll, "Dynamics, control and information in delay-coupled systems: An overview," Philos. Trans. Roy. Soc. London A: Math., Phys. Eng. Sci. 371, 20120465 (2013).

${ }^{31}$ I. Fischer, R. Vicente, J. M. Buldú, M. Peil, C. R. Mirasso, M. C. Torrent, and J. García-Ojalvo, "Zero-lag long-range synchronization via dynamical relaying," Phys. Rev. Lett. 97, 123902 (2006).

${ }^{32}$ D. V. R. Reddy, A. Sen, and G. L. Johnston, "Experimental evidence of time-delay-induced death in coupled limit-cycle oscillators," Phys. Rev. Lett. 85, 3381 (2000).
${ }^{33}$ R. Broom, E. Mohn, C. Risch, and R. Salathe, "Microwave selfmodulation of a diode laser coupled to an external cavity," IEEE J. Quantum Electron. 6, 328-334 (1970).

${ }^{34}$ J. P. Pade and T. Pereira, "Improving network structure can lead to functional failures," Sci. Rep. 5, 9968 (2015).

${ }^{35}$ J. D. Hart, J. P. Pade, T. Pereira, T. E. Murphy, and R. Roy, "Adding connections can hinder network synchronization of time-delayed oscillators," Phys. Rev. E 92, 022804 (2015).

${ }^{36} \mathrm{H}$. Sakaguchi and Y. Kuramoto, "A soluble active rotater model showing phase transitions via mutual entrainment,” Prog. Theor. Phys. 76, 576-581 (1986).

${ }^{37}$ N. Kopell and G. B. Ermentrout, "Coupled oscillators and the design of central pattern generators," Math. Biosci. 90, 87-109 (1988).

${ }^{38}$ E. M. Izhikevich, "Phase models with explicit time delays," Phys. Rev. E 58, 905 (1998).

${ }^{39}$ S. A. Campbell and I. Kobelevskiy, "Phase models and oscillators with time delayed coupling," Discrete Contin. Dyn. Syst. 8, 2653-2673 (2012).

${ }^{40}$ O. D'Huys, R. Vicente, T. Erneux, J. Danckaert, and I. Fischer, "Synchronization properties of network motifs: Influence of coupling delay and symmetry," Chaos: Interdiscip. J. Nonlinear Sci. 18, 037116 (2008).

${ }^{41}$ O. D'Huys, R. Vicente, J. Danckaert, and I. Fischer, "Amplitude and phase effects on the synchronization of delay-coupled oscillators," Chaos 20, 043127 (2010).

${ }^{42}$ R. Milo, S. Shen-Orr, S. Itzkovitz, N. Kashtan, D. Chklovskii, and U. Alon, "Network motifs: Simple building blocks of complex networks," Science 298, 824-827 (2002).

${ }^{43}$ U. Kim, M. V. Sanchez-Vives, and D. A. McCormick, "Functional dynamics of gabaergic inhibition in the thalamus," Science 278, 130-134 (1997).

${ }^{44}$ D. Debay, J. Wolfart, Y. Le Franc, G. Le Masson, and T. Bal, "Exploring spike transfer through the thalamus using hybrid artificial-biological neuronal networks," J. Physiol. Paris 98, 540-558 (2004).

${ }^{45}$ G. M. Shepherd, The Synaptic Organization of the Brain (Oxford University Press, 2003).

${ }^{46}$ G. B. Mindlin and R. Laje, The Physics of Birdsong (Springer Science \& Business Media, 2006).

${ }^{47}$ O. Sporns and R. Kötter, "Motifs in brain networks," PLoS Biol. 2, e369 (2004).

${ }^{48}$ J. Qian, A. Hintze, and C. Adami, "Colored motifs reveal computational building blocks in the c. elegans brain," PLoS One 6, e17013 (2011).

${ }^{49}$ E. M. Izhikevich, Dynamical Systems in Neuroscience: The Geometry of Excitability and Bursting (MIT Press, Cambridge, 2006).

${ }^{50}$ A. Pikovsky, M. Rosenblum, and J. Kurths, Synchronization: A Universal Concept in Nonlinear Sciences (Cambridge University Press, Cambridge, UK, 2001).

${ }^{51}$ A. L. Hodgkin and A. F. Huxley, "A quantitative description of membrane current and its application to conduction and excitation in nerve," J. Physiol. 117, 500-544 (1952).

${ }^{52}$ C. Koch, Biophysics of Computation (Oxford University Press, New York, 1999).

${ }^{53} \alpha_{n}(V)=\frac{10-V}{100\left(e^{10-V / 10}-1\right)}, \beta_{n}(V)=0.125 e^{-V / 80}, \alpha_{m}(V)=\frac{25-V}{10\left(e^{25-V) / 10}-1\right)}, \beta_{m}(V)$ $=4 e^{-V / 18}, \alpha_{h}(V)=0.07 e^{-V / 20}$, and $\beta_{h}(V)=\frac{1}{\left(e^{(30-V) / 10}+1\right)}$. Here, all voltages are measured in $\mathrm{mV}$, with the resting potential shifted to zero $\mathrm{mV}$.

${ }^{54}$ M. d S. Baptista, T. Pereira, and J. Kurths, "Upper bounds in phase synchronous weak coherent chaotic attractors," Phys. D: Nonlinear Phenom. 216, 260-268 (2006).

${ }^{55}$ T. Pereira, M. Baptista, and J. Kurths, "Phase and average period of chaotic oscillators," Phys. Lett. A 362, 159-165 (2007).

${ }^{56} \mathrm{H}$. Smith, An Introduction to Delay Differential Equations with Applications to the Life Sciences (Springer Science \& Business Media, 2010), Vol. 57.

${ }^{57}$ T. Pereira, J. Eldering, M. Rasmussen, and A. Veneziani, "Towards a theory for diffusive coupling functions allowing persistent synchronization," Nonlinearity 27, 501 (2014).

${ }^{58}$ M. G. Rosenblum, A. S. Pikovsky, and J. Kurths, "From phase to lag synchronization in coupled chaotic oscillators,” Phys. Rev. Lett. 78, 4193 (1997). 